\title{
Orthogonal Drawings of Plane Graphs without Bends (Extended Abstract)
}

\author{
Md. Saidur Rahman ${ }^{1}$, Mahmuda Naznin $^{1}$, and Takao Nishizeki ${ }^{2}$ \\ 1 Department of computer Science and Engineering, Bangladesh University of \\ Engineering and Technology (BUET), Dhaka-1000, Bangladesh. \\ saidur@cse.buet.edu, papri@cse.buet.edu \\ 2 Graduate School of Information Sciences, Tohoku University, Aoba-yama 05, \\ Sendai 980-8579, Japan. nishi@ecei.tohoku.ac.jp
}

\begin{abstract}
In an orthogonal drawing of a plane graph $G$ each vertex is drawn as a point and each edge is drawn as a sequence of vertical and horizontal line segments. A point at which the drawing of an edge changes its direction is called a bend. Every plane graph of the maximum degree at most four has an orthogonal drawing, but may need bends. A simple necessary and sufficient condition has not been known for a plane graph to have an orthogonal drawing without bends. In this paper we obtain a necessary and sufficient condition for a plane graph $G$ of the maximum degree three to have an orthogonal drawing without bends. We also give a linear-time algorithm to find such a drawing of $G$ if it exists.
\end{abstract}

Keywords: Graph, Algorithm, Graph Drawing, Orthogonal Drawing, Bend.

\section{Introduction}

Automatic graph drawings have numerous applications in VLSI circuit layout, networks, computer architecture, circuits schematics etc. For the last few years many researchers have concentrated their attention on graph drawings and introduced a number of drawing styles. Among these styles "orthogonal drawings" have attracted much attention due to their various applications, specially in circuit schematics, entity relationship diagrams, data flow diagrams etc. [DETT99]. An orthogonal drawing of a plane graph $G$ is a drawing of $G$ with the given embedding in which each vertex is mapped to a point, each edge is drawn as a sequence of alternate horizontal and vertical line segments, and any two edges do not cross except at their common end. A bend is a point where an edge changes its direction in a drawing. Every plane graph of the maximum degree four has an orthogonal drawing, but may need bends. For the cubic plane graph in Fig. 1(a) each vertex of which has degree 3, two orthogonal drawings are shown in Figs. 1(b) and (c) with 6 and 5 bends respectively. Minimization of 
the number of bends in an orthogonal drawing is a challenging problem. Several works have been done on this issue [GT95, GT97, RNN99, T87]. In particular, Garg and Tamassia [GT97] presented an algorithm to find an orthogonal drawing of a given plane graph $G$ with the minimum number of bends in time $O\left(n^{7 / 4} \sqrt{\log n}\right)$, where $n$ is the number of vertices in $G$. Rahman et al. gave an algorithm to find an orthogonal drawing of a given triconnected cubic plane graph with the minimum number of bends in linear time [RNN99].

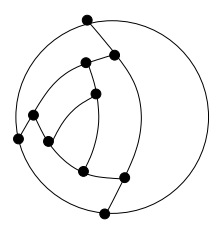

(a)

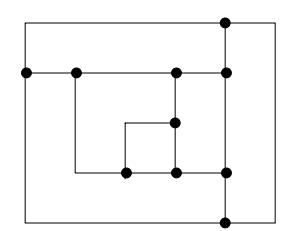

(b)

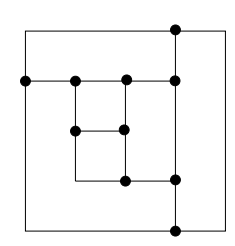

(c)

Fig. 1. (a) A plane graph $G$, (b) an orthogonal drawing of $G$ with 6 bends, and (c) an orthogonal drawing of $G$ with 5 bends.

In a VLSI floorplanning problem, an input is often a plane graph of the maximum degree 3 [L90, RNN00a, RNN00b]. Such a plane graph $G$ may have an orthogonal drawing without bends. The graph in Fig. 2(a) has an orthogonal drawing without bends as shown in Fig. 2(b). However, not every plane graph of the maximum degree 3 has an orthogonal drawing without bends. For example, the cubic plane graph in Fig. 1(a) has no orthogonal drawing without bends, since any orthogonal drawing of the outer cycle of the graph needs at least four bends. Thus one may assume that there are four or more vertices of degree two on the outer cycle of $G$. It is interesting to know which classes of such plane graphs have orthogonal drawings without bends. However, no simple necessary and sufficient condition has been known for a plane graph to have an orthogonal drawing without bends, although one can know in time $O\left(n^{7 / 4} \sqrt{\log n}\right)$ by the algorithm [GT97] whether a given plane graph has an orthogonal drawing without bends.

In this paper we obtain a simple necessary and sufficient condition for a plane graph $G$ of the maximum degree 3 to have an orthogonal drawing without bends. The condition leads to a linear-time algorithm to find an orthogonal drawing of $G$ without bends if it exists.

The rest of the paper is organized as follows. Section 2 describes some definitions and presents known results. Section 3 presents our results on orthogonal drawings of biconnected plane graphs without bends. Section 4 deals with orthogonal drawings of arbitrary (not always biconnected) plane graphs without bends. Finally Section 5 gives the conclusion. 


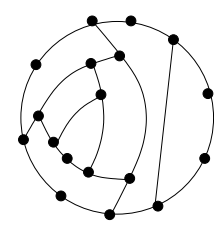

(a)

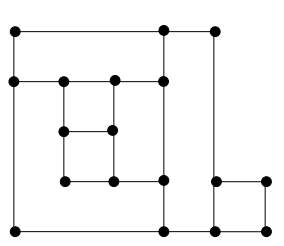

(b)

Fig. 2. (a) A plane graph $G$ and (b) an orthogonal drawing of $G$ without bends.

\section{Preliminaries}

In this section we give some definitions and preliminary known results.

Let $G$ be a connected simple graph with $n$ vertices and $m$ edges. We denote the set of vertices of $G$ by $V(G)$ and the set of edges by $E(G)$. The degree of a vertex $v$ is the number of neighbors of $v$ in $G$. We denote the maximum degree of graph $G$ by $\Delta(G)$ or simply by $\Delta$. The connectivity $\kappa(G)$ of a graph $G$ is the minimum number of vertices whose removal results in a disconnected graph or a single vertex graph. We say that $G$ is $k$-connected if $\kappa(G) \geq k$. We call a vertex of $G$ a cut vertex if its removal results in a disconnected graph.

A graph is planar if it can be embedded in the plane so that no two edges intersect geometrically except at a vertex to which they are both incident. A plane graph $G$ is a planar graph with a fixed planar embedding. A plane graph $G$ divides the plane into connected regions called faces. We refer the contour of a face as a clockwise cycle formed by the edges on the boundary of the face. We denote the contour of the outer face of $G$ by $C_{o}(G)$.

An edge of $G$ which is incident to exactly one vertex of a cycle $C$ and located outside $C$ is called a leg of the cycle $C$. The vertex of $C$ to which a leg is incident is called a leg-vertex of $C$. A cycle in $G$ is called a $k$-legged cycle of $G$ if $C$ has exactly $k$ legs in $G$.

An orthogonal drawing of a plane graph $G$ is a drawing of $G$ with the given embedding in which each vertex is mapped to a point, each edge is drawn as a sequence of alternate horizontal and vertical line segments, and any two edges do not cross except at their common end. A bend is a point where an edge changes its direction in a drawing. A rectangular drawing of a plane graph $G$ is a drawing of $G$ such that each edge is drawn as a horizontal or a vertical line segment, and each face is drawn as a rectangle. Thus a rectangular drawing is an orthogonal drawing in which there is no bends and each face is drawn as a rectangle. The following result is known on rectangular drawings.

Lemma 1. Let $G$ be a plane biconnected graph with $\Delta \leq 3$. Assume that four vertices of degree 2 on $C_{o}(G)$ are designated as the four corners of the outer rectangle. Then $G$ has a rectangular drawing if and only if $G$ satisfies the following two conditions [T84]: 
(r1) every 2-legged cycle contains at least two designated vertices, and (r2) every 3-legged cycle contains at least one designated vertex.

Furthermore one can check in linear time whether $G$ satisfies the condition above, and if $G$ does then one can find a rectangular drawing in linear time [RNN98].

A cycle in $G$ violating (r1) or (r2) is called a bad cycle: a 2-legged cycle is bad if it contains at most one designated vertex; a 3-legged cycle is bad if it contains no designated vertex.

A linear-time algorithm has been obtained in [RNN98] to find a rectangular drawing of a plane graph which has four designated corner vertices and satisfies the conditions in Lemma 1. We call it Algorithm Rectangular-Draw and use it in our orthogonal drawing algorithm in this paper.

For a cycle $C$ in a plane graph $G$, we denote by $G(C)$ the plane subgraph of $G$ inside $C$ (including $C$ ). A bad cycle $C$ in $G$ is called a maximal bad cycle if $G(C)$ is not contained in $G\left(C^{\prime}\right)$ for any other bad cycle $C^{\prime}$ of $G$. We say that cycles $C$ and $C^{*}$ in a plane graph $G$ are independent of each other if $G(C)$ and $G\left(C^{*}\right)$ have no common vertex. We now have the following lemma.

Lemma 2. Let $G$ be a biconnected plane graph of $\Delta \leq 3$, and let four vertices of degree 2 on $C_{o}(G)$ be designated as corners. Then the maximal bad cycles in $G$ are independent of each other.

\section{Orthogonal Drawings of Biconnected Plane Graphs}

In this section we present our results on orthogonal drawings of biconnected plane graphs. From now on we assume that $G$ is a biconnected plane graph with $\Delta \leq 3$ and there are four or more vertices of degree 2 on $C_{o}(G)$. The following theorem is the main result of this section.

Theorem 1. Let $G$ be a plane biconnected graph with $\Delta \leq 3$ and four or more vertices on $C_{o}(G)$. Then $G$ has an orthogonal drawing without bends if and only if any 2-legged cycle in $G$ contains at least two vertices of degree 2 and any 3legged cycle in $G$ contains at least one vertex of degree 2.

Note that Theorem 1 is a generalization of Lemma 1

It is easy to prove the necessity of Theorem 1 as follows.

Necessity of Theorem 1. Assume that a plane biconnected graph $G$ has an orthogonal drawing $D$ without bends.

Let $C$ be any 2-legged cycle. Then the drawing of $C$ in $D$ has at least four convex corners (of interior angle $90^{\circ}$ ). These convex corners must be vertices since $D$ has no bends. The two leg-vertices of $C$ may serve as two of the convex corners. However, each of the other convex corners must be a vertex of degree 2. Thus $C$ must contain at least two vertices of degree 2 .

Similarly we can show that any 3 -legged cycle $C$ in $G$ contains at least one vertex of degree 2 . 
In the rest of this section we give a constructive proof for the sufficiency of Theorem 1 and show that the proof leads to a linear-time algorithm to find an orthogonal drawing of a plane biconnected graph without bends if it exists.

Assume that $G$ satisfies the condition in Theorem 11. We now need some definitions. Let $C$ be a 2-legged cycle in $G$, and let $x$ and $y$ be the two leg vertices of $C$. We say that an orthogonal drawing $D(G(C))$ of the subgraph $G(C)$ is feasible if $D(G(C))$ has no bend and satisfies the following condition (f1) or (f2).

(f1) The drawing $D(G(C))$ intersects neither the first quadrant with the origin at $x$ nor the third quadrant with the origin at $y$ (after rotating the drawing and renaming the leg-vertices if necessary). (See Fig. 3.) Note that $C$ is not always drawn by a rectangle.
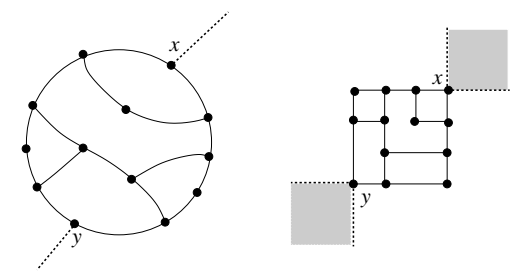

Fig. 3. Illustration of (f1) for a 2-legged cycle.

(f2) The drawing $D(G(C))$ intersects neither the first quadrant with the origin at $x$ nor the fourth quadrant with the origin at $y$ (after rotating the drawing and renaming the leg-vertices if necessary). (See Fig. 4.)
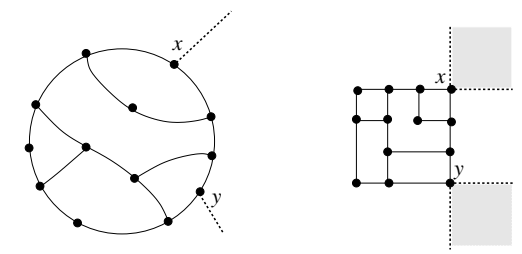

Fig. 4. Illustration of (f2) for a 2-legged cycle.

Let $C$ be a 3-legged cycle in $G$, and let $x, y$ and $z$ be the three leg-vertices. One may assume that $x, y$ and $z$ appear clockwise on $C$. We say that an orthogonal 
drawing $D(G(C))$ of $G(C)$ is feasible if $D(G(C))$ has no bend and $D(G(C))$ satisfies the following condition (f3).

(f3) The drawing $D(G(C))$ intersects none of the following three quadrants: the first quadrant with origin at $x$, the fourth quadrant with origin at $y$, and the third quadrant with origin at $z$ (after rotating the drawing and renaming the leg-vertices if necessary). (See Fig. [5])

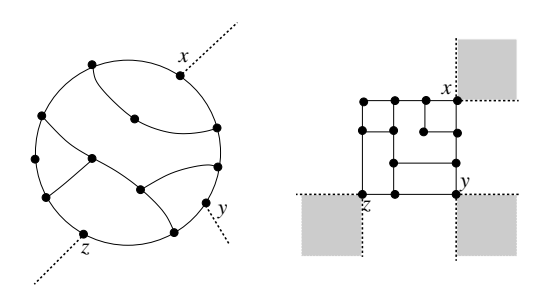

Fig. 5. Illustration of (f3) for a 3-legged cycle.

The conditions (f1), (f2) and (f3) imply that, in the drawing of $G(C)$, any vertex of $G(C)$ except leg-vertices is located in none of the shaded quadrants in Figs. 3, 4 and 15, and hence a leg incident to $x, y$ or $z$ can be drawn by a horizontal or a vertical line segments without edge-crossing as indicated by dotted lines in Figs. 3. 4 and [5.

We now have the following lemma.

Lemma 3. Let $G$ be a plane biconnected graph with $\Delta \leq 3$ and four or more vertices on $C_{o}(G)$, and assume that $G$ satisfies the condition in Theorem 1 , that is, any 2-legged cycle in $G$ contains at least two vertices of degree 2 and any 3-legged cycle in $G$ contains at least one vertex of degree 2. Then $G(C)$ has a feasible orthogonal drawing for any 2- or 3-legged cycle $C$ in $G$.

Proof. We give a recursive algorithm to find a feasible orthogonal drawing of $G(C)$. There are two cases to be considered.

Case 1: $C$ is a 2-legged cycle.

Let $x$ and $y$ be the two leg-vertices of $C$, and let $e_{x}$ and $e_{y}$ be the legs incident to $x$ and $y$, respectively. Since $C$ satisfies the condition in Theorem $\square C$ has at least two vertices of degree 2. Let $a$ and $b$ be any two vertices of degree 2 on $C$. We now regard the four vertices $x, y, a$ and $b$ as the four designated corner vertices of $C$.

We first consider the case where $G(C)$ has no bad cycle with respect to the four designated vertices. In this case, by Lemma $1 G(C)$ has a rectangular drawing $D$ with the four designated corner vertices. Such a rectangular drawing $D$ of $G(C)$ can be found by the algorithm Rectangular-Draw in [RNN98]. Since the 
outer cycle $C$ of $G(C)$ is drawn as a rectangle in $D, D$ satisfies Condition (f1) or (f2) and, in particular, $x, y, a$ and $b$ are the convex corners of the rectangular drawing of $C$. Since $D$ is a rectangular drawing, $D$ has no bend. Thus $D$ is a feasible orthogonal drawing of $G(C)$.

We then consider the case where $G(C)$ has a bad cycle. Let $C_{1}, C_{2}, \cdots, C_{l}$ be the maximal bad cycles of $G(C)$. By Lemma $2 C_{1}, C_{2}, \cdots, C_{l}$ are independent of each other. Construct a plane graph $Q$ from $G(C)$ by contracting $G\left(C_{i}\right), 1 \leq$ $i \leq l$, to a single vertex $v_{i}$, as illustrated in Figs. 6(a) and (b). Clearly $Q$ is a plane biconnected graph with $\Delta \leq 3$. Every bad cycle $C_{i}$ in $G(C)$ contains at most one designated vertex. If $C_{i}$ contains a designated vertex, then we newly designate $v_{i}$ as a corner vertex of $Q$ in place of the designated vertex. Thus $Q$ has exactly four designated vertices. (In Fig. [6 $Q$ has four designated vertices $a$, $b, x$, and $v_{2}$ since the bad cycle $C_{2}$ contains $y$.) Since all maximal bad cycles are contracted to single vertices in $Q, Q$ has no bad cycle with respect to the four designated vertices, and hence $Q$ has a rectangular drawing $D(Q)$, as illustrated in Fig. 6(c). Such a drawing $D(Q)$ can be found by Algorithm RectangularDraw. Clearly there is no bend on $D(Q)$. The shrunken outer cycle of $G(C)$ is drawn as a rectangle in $D(Q)$, and hence $D(Q)$ satisfies conditions (f1) or (f2). If $C_{i}$ is a 2-legged cycle, then $v_{i}$ and the two legs $e_{x_{i}}$ and $e_{y_{i}}$ are embedded in $D(Q)$ as illustrated in Figs. 7(b) and 8(b) or as in their rotated ones, and $C_{i}$ and the two legs $e_{x_{i}}$ and $e_{y_{i}}$ have the embeddings in Figs. 7)(c) and 8)(c) and their rotated ones. If $C_{i}$ is a 3 -legged cycle, then $v_{i}$ and the three legs $e_{x_{i}}, e_{y_{i}}$ and $e_{z_{i}}$ are embedded in $D(Q)$ as illustrated in Fig. 9(b) or as in their rotated ones, and $C_{i}$ and three legs $e_{x_{i}}, e_{y_{i}}$ and $e_{z_{i}}$ have the embeddings in Fig. 9(c) and their rotated ones. One can obtain a drawing $D(G(C))$ of $G(C)$ from the drawings of $Q$ and $G\left(C_{i}\right) 1 \leq i \leq l$, as follows. Replace each $v_{i}, 1 \leq i \leq l$, in $D(Q)$ with one of the feasible embeddings of $G\left(C_{i}\right)$ in Fig. 77(c), Fig. 8] c) and Fig. 9(c) and their rotated one that corresponds to the embedding of $v_{i}$ with legs in $D(Q)$, and draw each leg of $C_{i}$ in $D(G(C))$ by a straight line segment having the same direction as the leg in $D(Q)$, as illustrated in Fig. 6(d). We call this operation a patching operation.

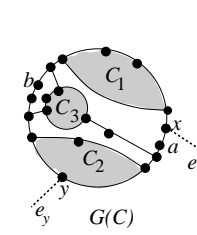

(a)

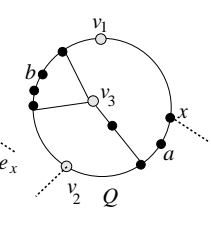

(b)

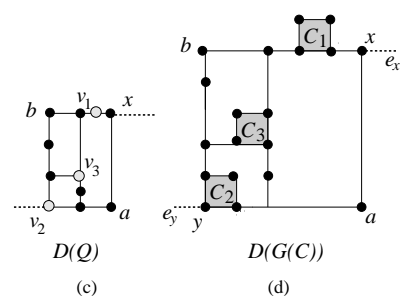

Fig. 6. Illustration for Case 1 where $C$ has the maximal bad cycles $C_{1}, C_{2}$ and $C_{3}$. 


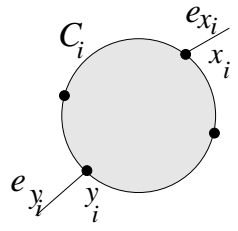

(a)

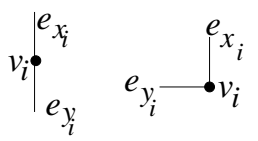

(b)

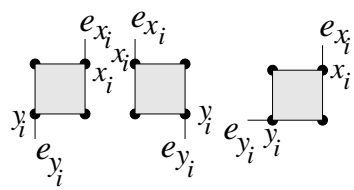

(c)

Fig. 7. (a) A 2-legged cycle $C_{i}$ having a feasible orthogonal drawing satisfying (f1), (b) embeddings of a vertex $v_{i}$ and two legs $e_{x_{i}}$ and $e_{y_{i}}$ incident to $v_{i}$, and (c) feasible orthogonal drawings of $G\left(C_{i}\right)$ with two legs.

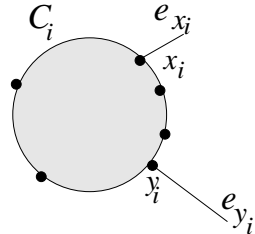

(a)

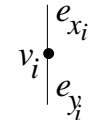

(b)

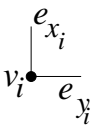

)

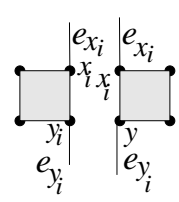

$e_{i}$

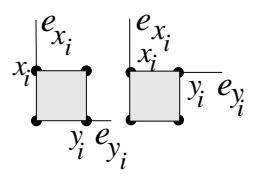

(c)

Fig. 8. (a) A 2-legged cycle $C_{i}$ having a feasible orthogonal drawing satisfying (f2), (b) embeddings of a vertex $v_{i}$ and two legs $e_{x_{i}}$ and $e_{y_{i}}$ incident to $v_{i}$, and (c) feasible orthogonal drawings of $G\left(C_{i}\right)$ with two legs.

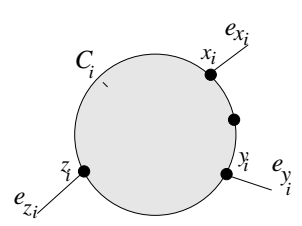

(a)

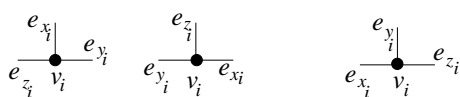

(b)

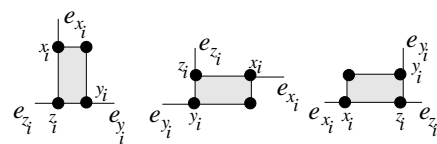

(c)

Fig. 9. (a) A 3-legged cycle $C_{i}$ having feasible orthogonal drawings satisfying (f3), (b) embeddings of a vertex $v_{i}$ and three legs $e_{x_{i}}, e_{y_{i}}$ and $e_{z_{i}}$ incident to $v_{i}$, and (c) feasible orthogonal drawings of $G\left(C_{i}\right)$ with three legs. 
We find a feasible orthogonal drawing $D\left(G\left(C_{i}\right)\right)$ of $G\left(C_{i}\right), 1 \leq$ $i \leq l$, in a recursive manner. We then patch the drawings $D\left(G\left(C_{1}\right)\right)$, $D\left(G\left(C_{2}\right)\right), \cdots, D\left(G\left(C_{l}\right)\right)$ into $D(Q)$ by patching operation. Since there is no bend in any of $D\left(G\left(C_{1}\right)\right), D\left(G\left(C_{2}\right)\right), \cdots, D\left(G\left(C_{l}\right)\right)$, there is no bend in the resulting drawing $D(G(C))$. Since the outer boundary of $D(Q)$ is a rectangle and the resulting drawing $D(G(C))$ always expands outwards, $D(G(C))$ satisfies (f1) or (f2). Hence $D(G(C))$ is a feasible orthogonal drawing.

Case 2: $C$ is a 3-legged cycle.

Let $x, y$ and $z$ be the three leg-vertices of $C$, and let $e_{x}, e_{y}$ and $e_{z}$ be the legs incident to $x, y$ and $z$, respectively. Since $C$ satisfies the condition in Theorem 1 $C$ has at least one vertex of degree 2 . Let $a$ be any vertex of degree 2 on $C$. We now regard the four vertices $x, y, z$ and $a$ as designated corner vertices.

We first consider the case where $G(C)$ has no bad cycle with respect to the four designated vertices. In this case by Lemma 1 $G(C)$ has a rectangular drawing $D$ with the four designated vertices. Such a rectangular drawing $D$ of $G(C)$ can be found by the algorithm Rectangular-Draw. Since the outer cycle $C$ of $G(C)$ is drawn as a rectangle in $D, D$ satisfies the condition (f3). Since $D$ is a rectangular drawing, $D$ has no bend. Thus $D$ is a feasible orthogonal drawing of $G(C)$.

We then consider the case where $G(C)$ has a bad cycle. Let $C_{1}, C_{2}, \cdots, C_{l}$ be the maximal bad cycles of $G(C)$. By Lemma $2 C_{1}, C_{2}, \cdots, C_{l}$ are independent of each other. Construct a plane graph $Q$ from $G(C)$ by contracting each subgraph $G\left(C_{i}\right), 1 \leq i \leq l$, to a single vertex $v_{i}$. Clearly $Q$ is a plane biconnected graph wih $\Delta \leq 3, Q$ has no bad cycle with respect to the four designated vertices, and hence $Q$ has a rectangular drawing $D(Q)$. Such a drawing can be found by Algorithm Rectangular-Draw. Clearly there is no bend on $D(Q)$. Since the outer cycle of $Q$ is drawn as a rectangle in $D(Q), D(Q)$ satisfies the condition (f3).

We then find a feasible orthogonal drawing $D\left(G\left(C_{i}\right)\right)$ of $G\left(C_{i}\right), 1 \leq i \leq l$, in a recursive manner, and patch the drawings $D\left(G\left(C_{1}\right)\right), D\left(G\left(C_{2}\right)\right), \cdots, D\left(G\left(C_{l}\right)\right)$ into $D(Q)$. Since there is no bend in any of $D\left(G\left(C_{1}\right)\right), D\left(G\left(C_{2}\right)\right), \cdots, D\left(G\left(C_{l}\right)\right)$, there is no bend in the resulting drawing $D(G(C))$. Since the outer boundary of $D(Q)$ is a rectangle and $D(G(C))$ expands outwards, $D(G(C))$ satisfies (f3). Thus $D(G(C))$ is a feasible orthogonal drawing of $G(C)$.

We call the algorithm for obtaining a feasible orthogonal drawing of $G(C)$ as described in the proof of Lemma 3 Algorithm Feasible-Draw. We now have the following lemma.

Lemma 4. Algorithm Feasible-Draw finds a feasible orthogonal drawing of $G(C)$ in time $O(n(G(C))$, where $n(G(C))$ is the number of vertices in $G(C)$.

We are now ready to prove the sufficiency of Theorem 1; we actually prove the following lemma.

Lemma 5. Let $G$ be a plane biconnected graph with $\Delta \leq 3$ and four or more vertices of degree 2 on $C_{o}(G)$. If $G$ satisfies the conditions in Theorem 1 , then $G$ has an orthogonal drawing without bends. 
Proof. Since there are four or more vertices of degree 2 on $C_{o}(G)$, we designate any four of them as (convex) corners.

Consider first the case where $G$ does not have any bad cycle with respect to the four designated (convex) corners. Then by Lemma 1 there is a rectangular drawing of $G$. The rectangular drawing of $G$ has no bends. Hence it is an orthogonal drawing $D(G)$ of $G$ without bends.

Consider next the case where $G$ has bad cycles. Let $C_{1}, C_{2}, \cdots, C_{l}$ be the maximal bad cycles in $G$. By Lemma $2 C_{1}, C_{2}, \cdots, C_{l}$ are independent of each other. We contract each $G\left(C_{i}\right), 1 \leq i \leq l$, to a single vertex $v_{i}$. Let $G^{*}$ be the resulting graph. Clearly, $G^{*}$ has no bad cycle with respect to the four designated vertices, some of which may be vertices resulted from the contraction of bad cycles. By Lemma $1 G^{*}$ has a rectangular drawing $D\left(G^{*}\right)$, which can be found by the algorithm Rectangular-Draw. We recursively find a feasible orthogonal drawing of each $G\left(C_{i}\right), 1 \leq i \leq l$, by Feasible-Draw. Patch the feasible orthogonal drawings of $G\left(C_{1}\right), G\left(C_{2}\right), \cdots, G\left(C_{l}\right)$ into $D\left(G^{*}\right)$ by patching operations. The resulting drawing is an orthogonal drawing $D$ of $G$. Note that $D\left(G^{*}\right)$ has no bend and $D\left(G\left(C_{i}\right)\right), 1 \leq i \leq l$, has no bend. Furthermore, patching operation introduces no new bend. Thus $D$ has no bend.

We call the algorithm for obtaining an orthogonal drawing of a biconnected plane graph $G$ described in the proof of Lemma 5 Algorithm Bi-OrthogonalDraw. We now have the following theorem.

Theorem 2. If $G$ is a plane biconnected graph with $\Delta \leq 3$, has four or more vertices of degree 2 on $C_{o}(G)$, and satisfies the condition in Theorem 1 , then Algorithm Bi-Orthogonal-Draw finds an orthogonal drawing of $G$ in linear time.

\section{Orthogonal Drawings of Arbitrary Plane Graphs}

In this section we extend our result on biconnected plane graphs in Theorem 1 to arbitrary (not always biconnected) plane graphs with $\Delta \leq 3$ as in the following theorem.

Theorem 3. Let $G$ be a plane graph with $\Delta \leq 3$. Then $G$ has an orthogonal drawing without bends if and only if every $k$-legged cycle $C$ in $G$ contains at least $4-k$ vertices having degree 2 in $G$ for any $k, 0 \leq k \leq 3$.

The proof for the necessity of Theorem 3 is similar to the proof for the necessity of Theorem 10 In the rest of this section we give a constructive proof for the sufficiency of Theorem 3 We need some definitions.

We may assume that $G$ is a plane connected graph of $\Delta \leq 3$. We call a subgraph $H$ of $G$ a biconnected component of $G$ if $H$ is a maximal biconnected subgraph of $G$. We call a single edge $(u, v)$ of $G$ together with the vertices $u$ and $v$ a weakly biconnected component of $G$ if either both $u$ and $v$ are cut vertices or one of $u$ and $v$ is a cut vertex and the other one is a vertex of degree one. 
Let $C$ be a cycle in $G$, and let $v$ be a cut vertex of $G$ on $C$. We call $v$ an out-cut vertex for $C$ if $v$ is a leg-vertex of $C$ in $G$, otherwise we call $v$ an in-cut vertex for $C$. Any in-cut vertex for $C$ is not a convex corner (having interior angle $90^{\circ}$ ) of the drawing of $C$ in any orthogonal drawing of $G$; otherwise, the edge of $G$ which is incident to $v$ and is not on $C$ could not be drawn as a horizontal or vertical line segment. Similarly, any out-cut vertex for $C$ is not a concave corner (having interior angle $270^{\circ}$ ). Thus the orthogonal drawing of $G$ must satisfy the following condition (f4).

(f4) Every in-cut vertex for any cycle is not a convex corner and every out-cut vertex is not a concave corner in the drawing of the cycle.

We now have the following lemmas.

Lemma 6. Let $G$ be a connected plane graph of $\Delta \leq 3$ satisfying the condition in Theorem 3. Then any biconnected component $H$ of $G$ has an orthogonal drawing which has no bends and satisfies ( $\left.f_{4}\right)$.

We call two subgraphs $H_{i}$ and $H_{j}$ of $G$ are disjoint with each other if $H_{i}$ and $H_{j}$ have no common vertex. One can easily observe the following lemma.

Lemma 7. Let $G$ be a connected plane graph of $\Delta \leq 3$. Then the biconnected components in $G$ are disjoint with each other.

A block of a connected graph $G$ is either a biconnected component or a weakly biconnected component of the graph. The blocks and cut-vertices in a connected graph $G$ can be represented by a tree which is called the $B C$-tree of $G$. In the $B C$-tree of $G$ every block is represented by a $B$-node and each cut vertex of $G$ is represented by a $C$-node. The $B C$-tree of the plane graph $G\left(C_{1}\right)$ is depicted in Fig. 10(b), where each $B$-node is represented by a rectangle and each $C$-node is represented by a circle.

We call a cycle $C$ in $G$ a maximal cycle of $G$ if $G(C)$ is not contained in $G\left(C^{\prime}\right)$ for any other cycle $C^{\prime}$ in $G$. Thus a maximal cycle is an outer cycle of a biconnected component of $G$. The graph $G$ in Fig. 10)(a) has two maximal cycles $C_{1}$ and $C_{2}$ drawn by thick lines. $G(C)$ is called a maximal closed subgraph of $G$ if $C$ is a maximal cycle of $G$. We now have the following lemma.

Lemma 8. Let $G$ be a connected plane graph of $\Delta \leq 3$ satisfying the condition in Theorem 3 and let $C$ be a maximal cycle in $G$. Then $G(C)$ has an orthogonal drawing which has no bends and satisfies (f4).

Proof. We give an algorithm for finding an orthogonal drawing of $G(C)$ which has no bends and satisfies (f4).

If $G(C)$ is a biconnected component of $G$, then by Lemma $6 G(C)$ has an orthogonal drawing which has no bends and satisfies (f4). One may thus assume that $G(C)$ is not a biconnected component of $G$. Then $G(C)$ has some biconnected components and weakly biconnected components. By Lemma 7 the biconnected components of $G(C)$ are disjoint with each other. We can find an 
orthogonal drawing of a biconnected component which has no bend and satisfies (f4) by an algorithm similar to Algorithm Bi-Orthogonal-Draw. We can draw a weakly biconnected component by a horizontal or vertical line segment. It is thus remained to merge the drawings of biconnected components and weakly biconnected components without introducing new bends and edge crossings.

We construct a $B C$-tree of $G(C)$. Let $B_{0}$ be the node in the BC-tree corresponding to the biconnected component of $G(C)$ whose outer cycle is $C$. We consider the BC-tree of $G(C)$ as a rooted tree and regard $B_{0}$ as the root. Starting from the root we visit the tree by depth-first search and merge the orthogonal drawings of the blocks in the depth first-search order. Let $B_{0}, B_{1}, B_{2}, \cdots, B_{b}$ be the ordering of the blocks following a depth-first search order starting from $B_{0}$. The BC-tree of $G\left(C_{1}\right)$ of $G$ in Fig. 10/a) is depicted in Fig.10(b), where $B_{0}$ is the root of the tree and the other $B$-nodes are numbered according to a depth-first search order starting from $B_{0}$.

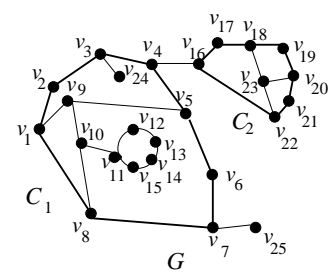

(a)

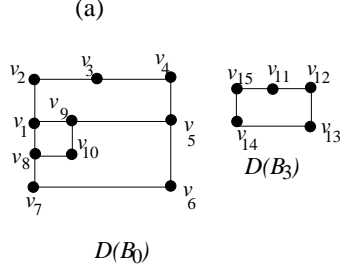

$D\left(B_{0}\right)$

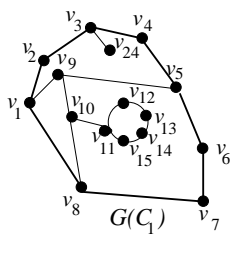

(b)

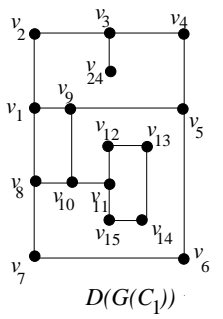

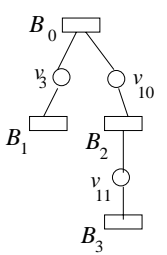
.

.


in the BC-tree. Then both $B_{x}$ and $B_{i+1}$ contain the vertex $v_{t}$, and $D_{i}$ contains the drawing of $B_{x}$. We have the following three cases to consider.

Case 1: $B_{x}$ is a biconnected component and $B_{i+1}$ is a weakly biconnected component.

In this case $B_{i+1}$ is an edge and will be drawn inside an inner face of the drawing $D_{i}$. Let $C_{f}$ be the facial cycle of $B_{x}$. Then $v_{t}$ is an in-cut vertex for $C_{f}$. Since we have obtained a feasible orthogonal drawing of $B_{x}$ which has no bends and satisfies (f4), $v_{t}$ is not drawn as convex corner in the drawing of $C_{f}$ in $D\left(B_{x}\right)$, and hence the embedding of $v_{t}$ in $D_{i}$ is one of the two embeddings in Fig. 11 or a rotated one. We can draw $B_{i+1}$ as a horizontal or a vertical line segment started from $v_{t}$ as illustrated by dotted lines in Fig. 11. Thus we obtain the drawing $D_{i+1}$. Clearly no new bend is introduced in $D_{i+1}$ and $D_{i}$ may be expanded outwards to avoid edge crossings. In Fig.10(c) the weakly biconnected component $B_{1}$ of edge $\left(v_{3}, v_{24}\right)$ is merged to a biconnected component $B_{0}$ at vertex $v_{3}$.

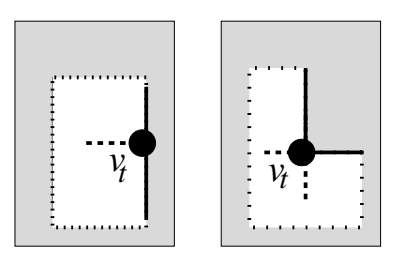

Fig. 11. Embeddings of $v_{t}$ in $D_{i}$ when $B_{x}$ is a biconnected component and $B_{i+1}$ is a weakly biconnected component.

Case 2: Both $B_{x}$ and $B_{i+1}$ are weakly biconnected components.

In this case $v_{t}$ is drawn in an inner face of $D_{i}$ and has degree 1 or 2 in $D_{i}$.

We first consider the case where $v_{t}$ has degree 1 . Then $v_{t}$ in $D_{i}$ has the embedding in Fig. 12(a) or a rotated one. We draw $B_{i}$ as the dotted line in Fig. [12(a).

We next consider the case where $v_{t}$ has degree 2 in $D_{i}$. Then $v_{t}$ has degree 3 in $G(C)$, and let $x, y$, and $z$ be the three neighbors of $v_{t}$ in $G$. We may assume without loss of generality that edges $\left(v_{t}, x\right)$ and $\left(v_{t}, y\right)$ are already drawn in $D_{i}$ and we now merge the drawing of the edge $\left(v_{t}, z\right)=B_{i+1}$ to $D_{i}$. It is evident from the drawing described above that $\left(v_{t}, x\right)$ and $\left(v_{t}, y\right)$ are drawn on a (horizontal or vertical) straight line segment. We draw the edge $\left(v_{t}, z\right)$ as a dotted line as in Fig. 12(b).

Case 3: $B_{x}$ is a weakly biconnected component and $B_{i+1}$ is a biconnected component.

In this case $v_{t}$ is drawn in $D_{i}$ as the end of a horizontal or vertical line segment inside an inner face of $D_{i}$. Vertex $v_{t}$ has degree 2 in $B_{i+1}$ and is an 


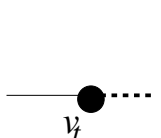

(a)

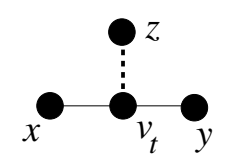

(b)

Fig. 12. Embedding of $B_{i}$ when both $B_{x}$ and $B_{i+1}$ are weakly biconnected components

out-cut vertex for $C_{o}\left(B_{i+1}\right)$. Hence by Lemma $6 v_{t}$ is not a concave corner of the drawing of $C_{o}\left(B_{i+1}\right)$ in $D\left(B_{i+1}\right)$. Therefore $D\left(B_{i+1}\right)$ can be easily merged with $D_{i}$ by rotating $D\left(G\left(B_{i+1}\right)\right) 90^{\circ}$ or $180^{\circ}$ or $270^{\circ}$ and expanding the drawing $D_{i}$ if necessary. In Fig. 10(c) the orthogonal drawing of $B_{3}$ is merged to $D_{2}$ at vertex $v_{11}$ where $D\left(B_{3}\right)$ has been rotated $90^{\circ}$ and the drawing $D_{2}$ is expanded outwards.

We call the algorithm described in the proof of Lemma 8 Algorithm

\section{Maximal-Orthogonal-Draw}

We are now ready to give a proof for the sufficiency of Theorem 3 .

Proof for Sufficiency of Theorem 3

We decompose $G$ into maximal closed subgraphs and weakly biconnected components. We find an orthogonal drawing of each maximal closed subgraph by Algorithm Maximal-Orthogonal-Draw. Each weakly biconnected component can be drawn by a horizontal or a vertical line segment. Using a technique similar to one in the proof of Lemma 8 we merge the drawings of maximal closed subgraphs and weakly biconnected components in the outer faces of maximal closed subgraphs. The resulting drawing is an orthogonal drawing of $G$ without bends.

We call the algorithm described in the proof for the sufficiency of Theorem 3 Algorithm No-bend-Orthogonal-Draw. We now have the following theorem.

Theorem 4. If $G$ is a plane connected graph of $\Delta \leq 3$ and satisfies the condition in Theorem [3, then Algorithm No-bend-Orthogonal-Draw finds an orthogonal drawing of $G$ without bends in linear time.

\section{Conclusions}

In this paper we established a necessary and sufficient condition for a plane graph $G$ with the maximum degree at most 3 to have an orthogonal drawing without bends. We gave a linear-time algorithm to determine whether $G$ has an orthogonal drawing without bends and find such a drawing of $G$ if it exists. It is remained as a future work to establish a necessary and a sufficient condition for a plane graph of the maximum degree at most 4 to have an orthogonal drawing without bends. 
Acknowledgment. We thank Prof. Shin-ichi Nakano for his comments and suggestions on a preliminary version of this paper.

\section{References}

[DETT99] G. Di. Battista, P. Eades, R. Tamassia, I. G. Tollis, Graph Drawing: Algorithms for the Visualization of Graphs, Prentice-Hall Inc., Upper Saddle River, New Jersey, 1999.

[GT95] A. Garg and R. Tamassia, On the computational complexity of upward and rectilinear planarity testing, Proc. of Graph Drawing'94, Lect. Notes in Computer Science, 894, pp. 99-110, 1995.

[GT97] A. Garg and R. Tamassia, A new minimum cost flow algorithm with applications to graph drawing, Proc. of Graph Drawing'96, Lect. Notes in Computer Science, 1190, pp. 201-206, 1997.

[L90] T. Lengauer, Combinatorial Algorithms for Integrated Circuit Layout, Wiley, Chichester, 1990.

[RNN00a] M. S. Rahman, S. Nakano and T. Nishizeki, Box-rectangular drawings of plane graphs, Journal of Algorithms, 37, pp. 363-398, 2000.

[RNN00b] M. S. Rahman, S. Nakano and T. Nishizeki, Rectangular drawings of plane graphs without designated corners, Proc. of COCOON'2000, Lect. Notes in Computer Science, 1858, pp. 85-94, 2000, also Comp. Geom. Theo. Appl., to appear.

[RNN98] M. S. Rahman, S. Nakano and T. Nishizeki, Rectangular grid drawings of plane graphs, Comp. Geom. Theo. Appl., 10(3), pp. 203-220, 1998.

[RNN99] M. S. Rahman, S. Nakano and T. Nishizeki, A linear algorithm for bendoptimal orthogonal drawings of triconnected cubic plane graphs, Journal of Graph Alg. and Appl., http://www.cs.brown.edu/publications/jgaa/, 3(4), pp. 31-62, 1999.

[T84] C. Thomassen, Plane representations of graphs, (Eds.) J.A. Bondy and U.S.R. Murty, Progress in Graph Theory, Academic Press Canada, pp. 43-69, 1984.

[T87] R. Tamassia, On embedding a graph in the grid with the minimum number of bends, SIAM J. Comput., 16, pp. 421-444, 1987. 